\title{
Characteristics improvement of top-gate self-aligned amorphous indium gallium zinc oxide thin-film transistors using a dual-gate control
}

\author{
Manoj Nag \\ Florian De Roose \\ Kris Myny \\ Soeren Steudel \\ Jan Genoe \\ Guido Groeseneken \\ Paul Heremans
}

\begin{abstract}
In this work, we have reported dual-gate amorphous indium gallium zinc oxide thin-film transistors (a-IGZO TFTs), where a top-gate self-aligned TFTs has a secondary bottom gate and the TFT integration comprises only five mask steps. The electrical characteristics of a-IGZO TFTs under different gate control are compared. With the enhanced control of the channel with two gates connected together, parameters such as on current $\left(\mathrm{I}_{\mathrm{ONN}}\right)$, sub-threshold slope $\left(\mathrm{SS}^{-1}\right)$, output resistance, and bias-stress instabilities are improved in comparison with single-gate control self-aligned a-IGZO TFTs. We have also investigated the applicability of the dual-gate a-IGZO TFTs in logic circuitry such as 19-stage ring oscillators.
\end{abstract}

Keywords - metal oxide, a-IGZO, TFT, self-aligned, dual gate, display technology.

DOI \# 10.1002/jsid.558

\section{Introduction}

In recent years, amorphous oxide semiconductors, particularly amorphous indium gallium zinc oxide (a-IGZO), have received great interest for thin-film transistor (TFT) applications. They have been investigated as an alternative to amorphous silicon and low-temperature polycrystalline silicon TFTs for active matrix liquid crystal displays and active matrix organic light emitting diode displays and also finding applications in the circuitry such as shift resistors, level shifters, buffers, drivers, and dc-dc converters. ${ }^{1-6}$ The aIGZO characteristics, such as high electron mobility, large area uniformity, and their ability to be fabricated at low temperatures on plastic substrates, are attractive for manufacturing the next generation large area display backplanes and circuits. For performance enhancement of the TFT-based electronic systems, high-speed operation is required. High mobility material and the channel length $(L)$ shrinking are the common way to improve the performance. In TFT configuration for high performance, coplanar selfaligned (SA) gate configuration with zero overlap capacitance (gate-source/drain overlap) is preferred compared with staggered bottom-gate (BG) (back-channel-etch and etchstop-layer) configuration. ${ }^{7-15}$ Another way to achieve the high performance is by the dual-gate (DG) control. Few groups have reported the realization of a-IGZO TFTs with DG control in both staggered (back-channel-etch and etch-stoplayer) and coplanar (standard and SA) configurations, where the top-gate (TG) and BG are electrically connected to each other. ${ }^{16-25}$ It is shown that much better control of the channel is achieved which resulted in improved characteristics, that is, higher apparent mobility, higher on-current $\left(\mathrm{I}_{\mathrm{ON}}\right)$, steeper sub-threshold slope $\left(\mathrm{SS}^{-1}\right)$, and a turn-on voltage $\left(\mathrm{V}_{\mathrm{ON}}\right)$ closer to zero volts. Most of the reported work in DG TFTs is with BG TFT structure where the parasitic capacitance issue due to gate and source/drain (S/D) overlap is not addressed. In other reported work with coplanar SA TG TFT structure, the total number of mask steps used are six or more, where two separated mask steps are required for contacting top gate and bottom gate. ${ }^{28}$ In this work, we have demonstrated SA TG a-IGZO TFTs with an additional BG using five mask steps only, thus reducing one mask step. The detailed improvement of the transfer characteristics, their uniformity (across the substrate and substrate to substrate), and channel length independence, $\mathrm{V}_{\mathrm{ON}}$ close to zero (the desirable parameters for the robust circuits), is demonstrated under DG operations.

\section{Experimental}

The schematic cross-sectional view of process integration and SEM image of DG TFT are shown in Fig. 1(a) and 1(b), respectively. In the first step, a buffer layer is deposited on the glass substrate. On the top of the buffer layer, a BG metal [MoCr deposited by physical vapor deposition (PVD)] is deposited and patterned. This is followed by deposition of first gate dielectric deposition $\left(\mathrm{SiO}_{2}\right.$ by plasma enhanced chemical vapor deposition (PECVD) at $350^{\circ} \mathrm{C}$ ). In the next

Received 12/31/16; accepted 04/27/17.

The authors are with imec, Kapeldreef 75, 3001 Leuven, Belgium; telephone +32-16-28-1700; e-mail: manoj.nag@imec.be.

Manoj Nag, Florian De Roose, Jan Genoe, Guido Groeseneken and Paul Heremans are with the ESAT, Katholieke Universiteit Leuven, Kasteelpark Arenberg 10, 3001 Leuven, Belgium.

Paul Heremans is with Holst Centre, High Tech Campus 31, 5656 AE Eindhoven, The Netherlands.

(C) Copyright 2017 Society for Information Display 1071-0922/17/0558\$1.00. 


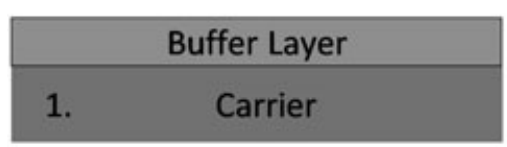

\#Mask 2 (a-IGZO)

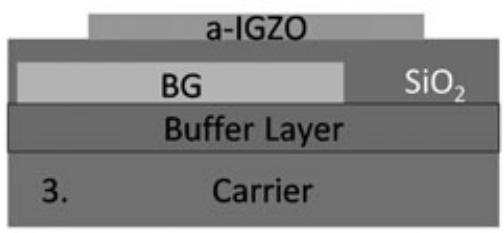

\#Mask 4 (Via)

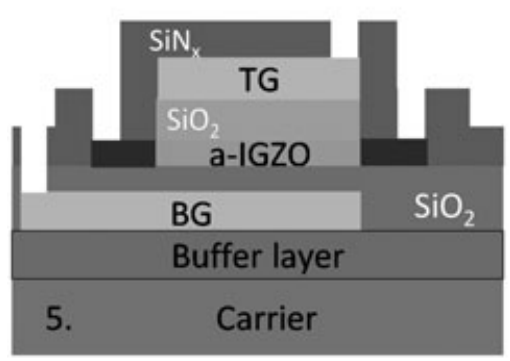

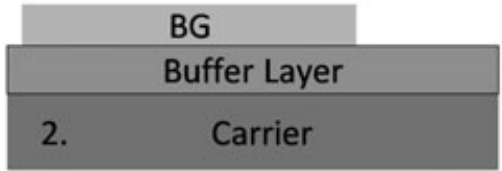

\#Mask 3 (Top-Gate)

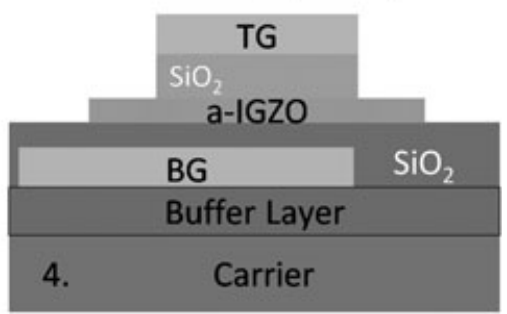

\#Mask 5 (Source/Drain)

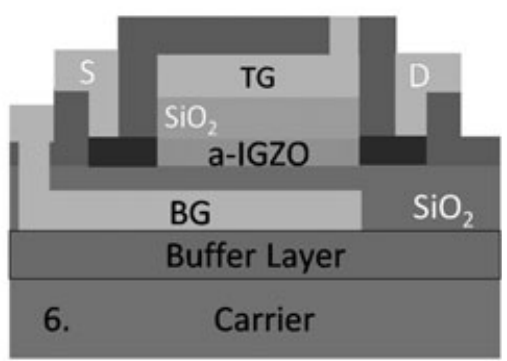

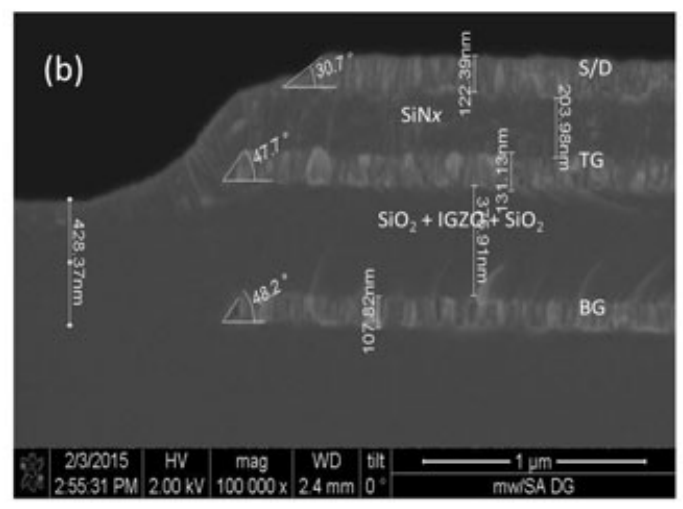

FIGURE 1 - (a) Cross-sectional view of the complete process flow and (b) final SEM view of dual-gate thin-film transistors. BG, bottom gate; TG, top gate.

step, a-IGZO layer $\left(20 \mathrm{~nm}\right.$ with $10 \%$ ratio of $\left.\mathrm{O}_{2} / \mathrm{Ar}\right)$ is deposited by de sputtering (PVD) and then patterned using wet etch. Furthermore, it is followed by the deposition of the second gate dielectric $\left(\mathrm{SiO}_{2}\right.$ deposition by PECVD at $250^{\circ} \mathrm{C}$ ) and the second gate metal layer $(\mathrm{MoCr})$. In the next step, the gate stack (metal and dielectric) was patterned using combined step of wet and dry etch. Subsequently, an interlayer dielectric $\left(\mathrm{SiN} x\right.$ by PECVD at $\left.250^{\circ} \mathrm{C}\right)$ is deposited. The contact vias are patterned in the next step using a dry etch process. This contact opening step is combined for contacting the BG metal, TG metal, and S/D regions (\#Mask 4). This saves one mask step compared with the earlier reported work on DG TFTs, ${ }^{27-35}$. In the last step, the S/D metal (Mo deposited by PVD) was deposited and then patterned using dry etch. Finally, the samples were annealed at $240^{\circ} \mathrm{C}$ in the $\mathrm{N}_{2}$ oven for $1 \mathrm{~h}$. All layers have been patterned using standard photolithography techniques, and all the processes were performed at a temperature below $350^{\circ} \mathrm{C}$ to enable integration on polyimide foil and a range of potential moisture barriers. ${ }^{26}$ The electrical characteristics of the individual TFTs and inverters were measured using a parameter analyzer (Agilent 4156) in $\mathrm{N}_{2}$ environment. The output characteristics of the ring oscillators were examined with an oscilloscope. 


\section{Results and discussion}

In coplanar SA TFT configuration, the sheet resistance $\left(\mathrm{R}_{\text {SHEET }}\right)$ of the spacing region (SP region between gate and $\mathrm{S} / \mathrm{D}$ as shown in Fig. 1(a)) and contact resistance $\left(\mathrm{R}_{\mathrm{CONTACT}}\right)$ are important factors. They are responsible for limiting the trans-conductance and hence the channel mobility. ${ }^{14,15} \mathrm{SiN} x$ interlayer (a-IGZO doping due to high hydrogen content of the layer) has been utilized to change the conductivity of the

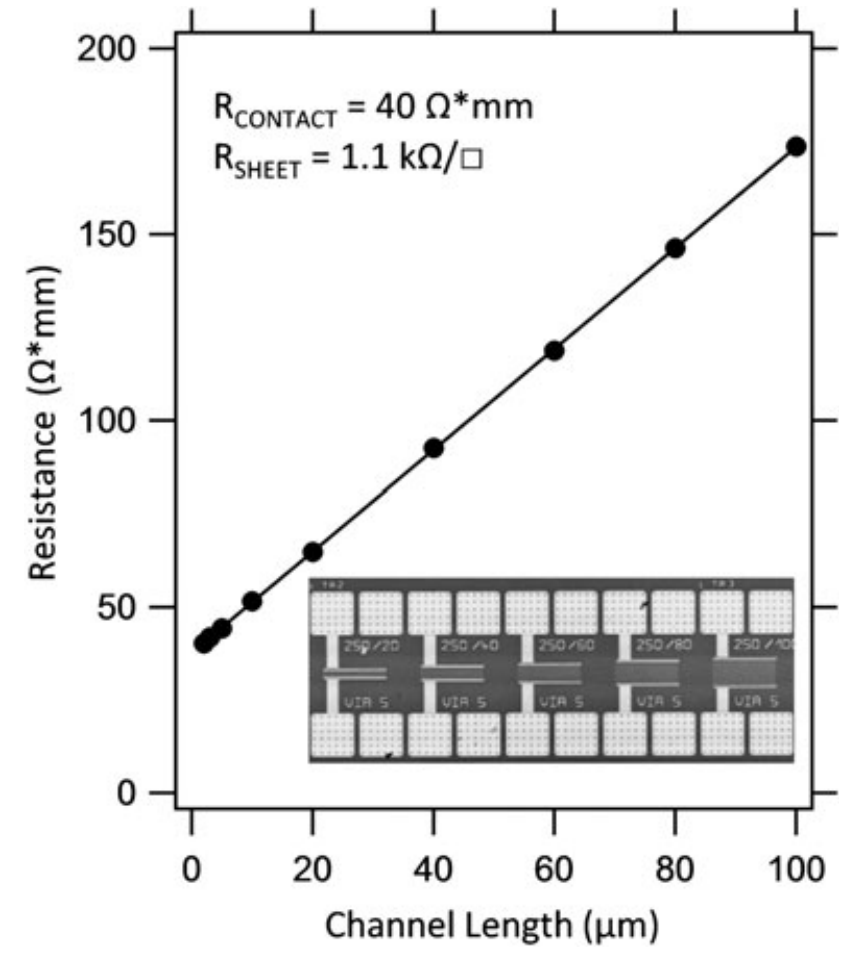

FIGURE 2 - The transistor resistance as a function of channel length ' $L$ ' for dual-gate thin-film transistors with $\mathrm{SiN} x$ interlayer. The contact resistances are obtained from the extrapolation to a zero channel length. The layout of contact layout is shown in the inset.
SP region. The contact resistance, $R_{\text {CONTACT }}=R_{S}+R_{D}$, with $R_{S}$ and $R_{D}$ respectively the source and drain resistances, and sheet resistance ( $\mathrm{R}_{\text {SHEET }}$ ) were extracted using the transfer length method. The utilized transfer length method structure contained nine TFTs with fixed channel width $(W=250 \mu \mathrm{m})$ and varying channel length $(L=10-100 \mu \mathrm{m})$ values from the width normalized contact resistance $\mathrm{R}_{\text {CONTACT }} W$ value versus channel length $L$ plot as shown in Fig. 2 . The R RHEET value of $\sim 1.1 \mathrm{k} \Omega / \square$ is obtained. This value is lower enough for the DG TFT's integration in our large-scale applications.

Typical transfer and output characteristics of the TFTs (W/ $L=15 / 5 \mu \mathrm{m} / \mu \mathrm{m}$ ) with TG, BG, and DG controls are shown in

TABLE 1 - Comparison of amorphous indium gallium zinc oxide thinfilm transistor characteristics at different operation conditions for $V_{G S}$ values between -20 and $20 \mathrm{~V}$ and $\mathrm{V}_{\mathrm{DS}}$ value of $20 \mathrm{~V}$.

\begin{tabular}{lcccc}
\hline Parameters & Bottom gate & Top gate & Dual gate & Isolated top gate \\
\hline$\mu_{\mathrm{FE}}\left(\mathrm{cm}^{2} / \mathrm{V} . \mathrm{s}\right)$ & 10.3 & 9.3 & 20.6 & 9.0 \\
$\mathrm{~V}_{\mathrm{ON}}(\mathrm{V})$ & -4.0 & -3.5 & 0 & -10.5 \\
$\mathrm{SS}^{-1}(\mathrm{~V} / \mathrm{dec})$ & 0.38 & 0.37 & 0.28 & 0.70 \\
$\mathrm{I}_{\mathrm{ON}} / \mathrm{I}_{\mathrm{OFF}}$ & $9.40 \times 10^{7}$ & $5.60 \times 10^{7}$ & $1.80 \times 10^{8}$ & $1.70 \times 10^{8}$ \\
\hline
\end{tabular}
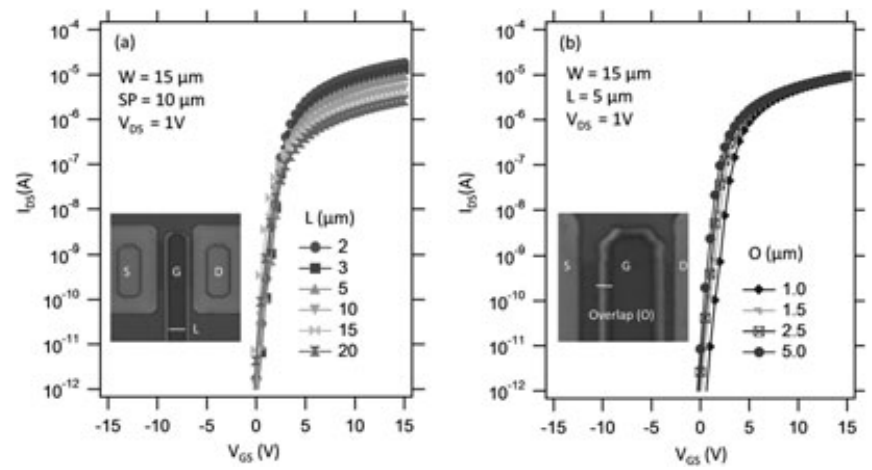

FIGURE 4 - Self-aligned thin-film transistor's transfer characteristics ( $V_{\mathrm{GS}}$ between -15 and $15 \mathrm{~V}$ and a $V_{D S}$ value of $1 \mathrm{~V}$ ) dependence on (a) channel length ' $L$ ' $(\mathrm{SP}=10 \mu \mathrm{m})$ and $(\mathrm{b})$ gate overlap 'O'.
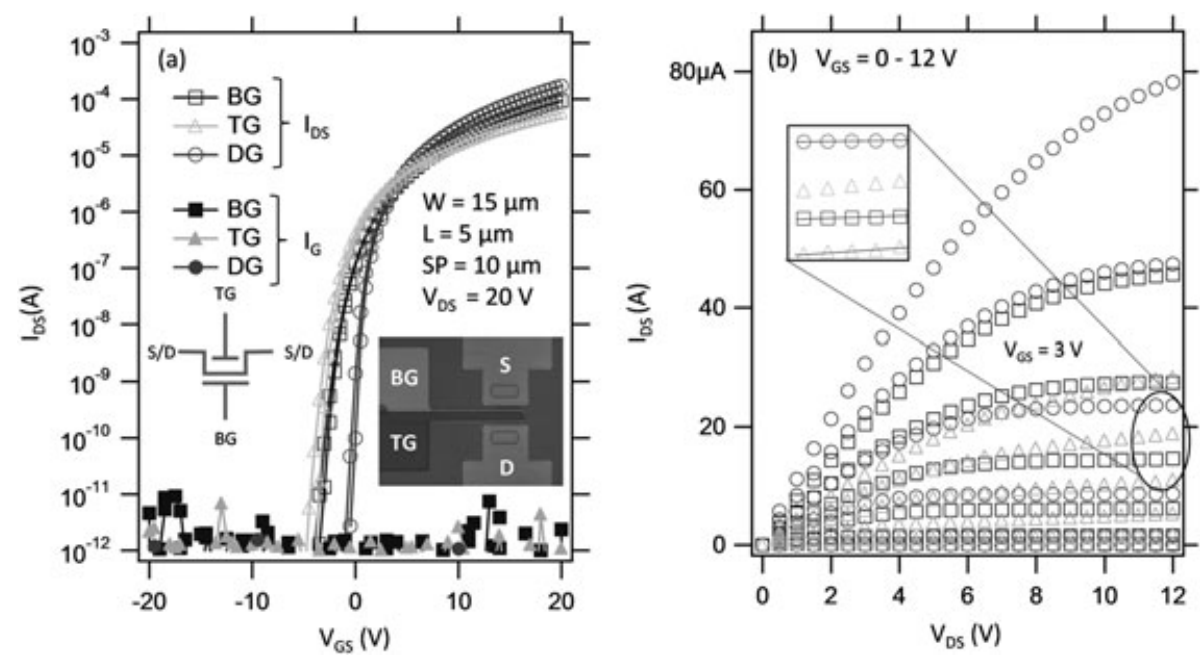

FIGURE 3 - (a) Transfer (at $V_{G S}$ between -20 and $20 \mathrm{~V}$ and a $V_{D S}$ value of $20 \mathrm{~V}$ ) and (b) output characteristics (at $\mathrm{V}_{\mathrm{DS}}$ of 0 to $12 \mathrm{~V}$ and $\mathrm{V}_{\mathrm{GS}}$ between 0 and $12 \mathrm{~V}$ ) of dual-gate thin-film transistors (DG TFTs). Layout of the thin-film transistor is shown in the inset. BG, bottom gate; TG, top gate. 

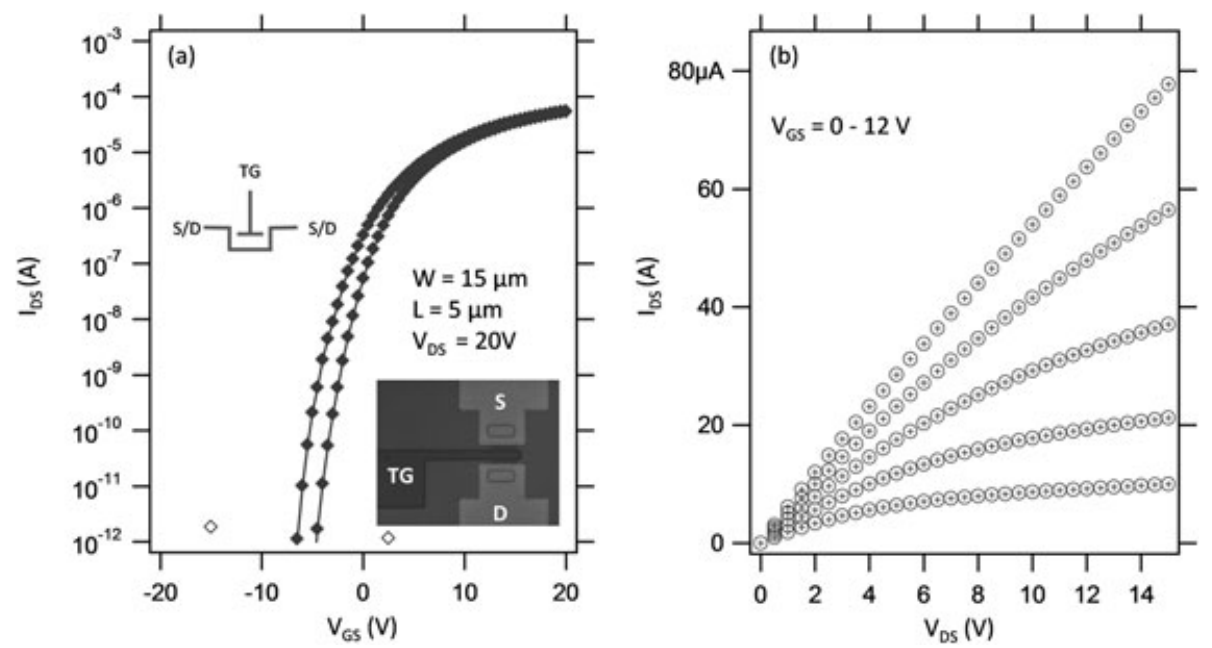

FIGURE 5 - (a) Transfer (at $V_{G S}$ between -20 and $20 \mathrm{~V}$ and a $V_{D S}$ value of $20 \mathrm{~V}$ ) and (b) output characteristics (at $V_{D S}$ of 0 to $12 \mathrm{~V}$ and $V_{G S}$ between 0 and $12 \mathrm{~V}$ ) of top-gate self-aligned thin-film transistors. Layout of the thin-film transistor is shown in the inset.
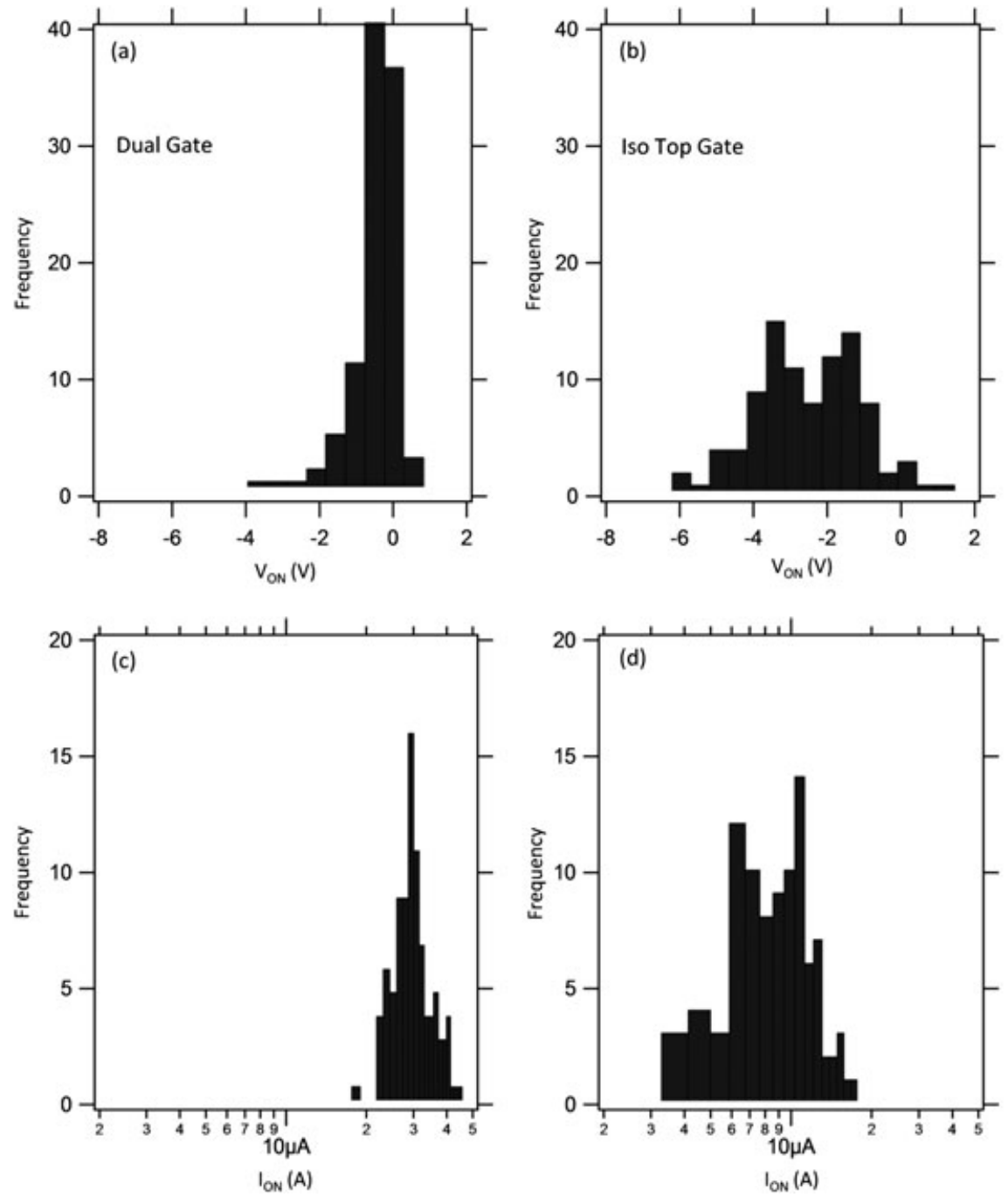

FIGURE 6 - Histogram of $\mathrm{V}_{\mathrm{ON}}$ and $\mathrm{I}_{\mathrm{ON}}$ for 100 amorphous indium gallium zinc oxide-isolated topgate and dual-gate thin-film transistors. 
Fig. 3(a) and 3(b), respectively. The microscope image of the TFT is also shown in Fig. 3(a) (inset view). Figures of merit of these TFTs at a drain-to-source voltage $\left(\mathrm{V}_{\mathrm{DS}}\right)$ of $20 \mathrm{~V}$ are summarized in Table 1. The DG TFTs show apparent mobility $\left(\mu_{\mathrm{FE}}\right)$ of $20.0 \mathrm{~cm}^{2} / \mathrm{V} . \mathrm{s}, \mathrm{SS}^{-1}$ of $0.28 \mathrm{~V} /$ decade, and current $\left(\mathrm{I}_{\mathrm{ON}} /\right.$ $\left.\mathrm{I}_{\mathrm{OFF}}\right)$ ratio of $1.8 \times 10^{8}$. They exhibit over three times increase in $\mathrm{I}_{\mathrm{ON}}$ and one and a half time steeper value of $\mathrm{SS}^{-1}$ when compared with single (top) gate TFTs. The turn-on-voltage $\left(\mathrm{V}_{\mathrm{ON}}\right)$ value of close to zero volts compare with single-gated TFTs is another improvement. The gate leakage current caused by the increased gate area is negligible as well. In output characteristics, no s-shaped behavior is observed at

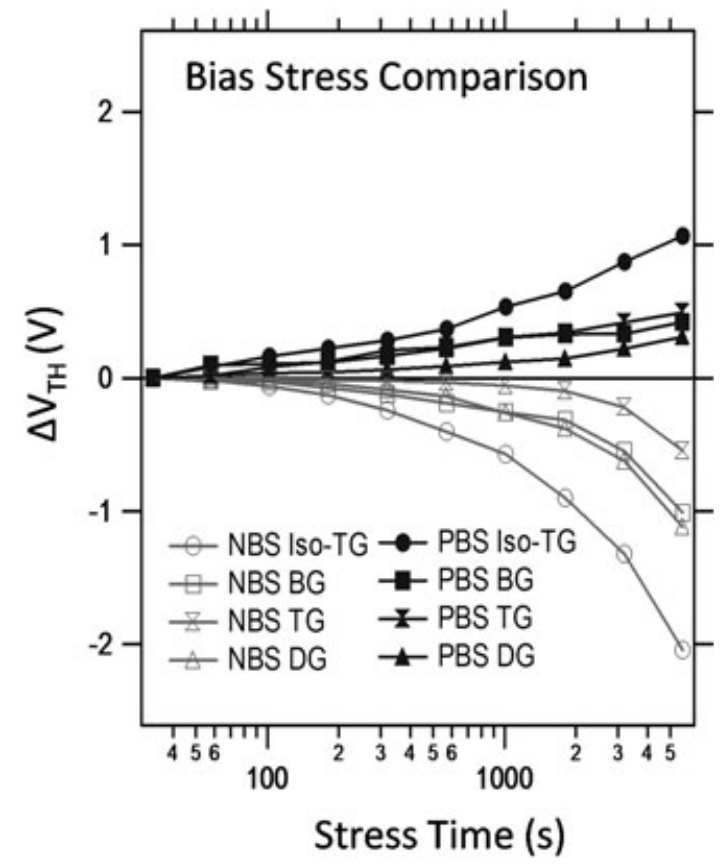

FIGURE $7-V_{\mathrm{TH}}$ shift as function of stress time under $+1 \mathrm{MV} / \mathrm{cm}^{\left(\mathrm{GS}^{-}\right.}$ $\mathrm{V}_{\mathrm{ON}}=+20 \mathrm{~V}$ and $\left.\mathrm{V}_{\mathrm{DS}}=0 \mathrm{~V}\right)$ and under $1 \mathrm{MV} / \mathrm{cm}\left(\mathrm{V}_{\mathrm{GS}}-\mathrm{V}_{\mathrm{ON}}= \pm 20 \mathrm{~V}\right.$ and $V_{D S}=0 \mathrm{~V}$ ) field strength in dark at room temperature. low values of $V_{D S}$. This means that the $S / D$ regions are nicely doped. Further, the output resistance (change in $\mathrm{I}_{\mathrm{DS}}$ /change in $\mathrm{V}_{\mathrm{DS}}\left(\Delta \mathrm{I}_{\mathrm{DS}} / \Delta \mathrm{V}_{\mathrm{DS}}\right)$ at $\left.\mathrm{V}_{\mathrm{GS}}=3.0 \mathrm{~V}\right)$ is low for the DG TFTs in comparison with the independent TG and BG TFTs. All these improvements are due to the improved control of the channel potential when the two gates are connected together. Furthermore, to verify again that the $\mathrm{R}_{\text {SHEET }}$ of the gatesource contact is well distributed across the channel spacing and not influencing the channel length, the transfer characteristics of DG TFTs (at $\mathrm{V}_{\mathrm{DS}}=1 \mathrm{~V}$ ) with different channel lengths $(L=2,3,5,10$, and $20 \mu \mathrm{m})$ were compared. The characteristic such as $I_{O N}$ nicely scale with channel length is shown in Fig. 4(a). In another comparison, TFTs with different overlaps $(\mathrm{O}=1.0,1.5,2.0$, and $5 \mu \mathrm{m}$, where $\mathrm{O}$ is defined as the difference between the channel lengths of the bottom and the top gate) were compared. It is to be noticed that with overlap change, the space region does change between the gate and S/D. This could possibly impact the TFT characteristics if the doping of the space region is not uniform. As shown in Fig. 4(b), no variation of TFT characteristics with different gate overlaps is observed. This clarifies that the spacing region is uniformly doped.

It is also worth mentioning that the improved metallic shielding reduces the impact of buffer layer out-degassing ${ }^{27}$ on the TFT characteristics because the isolated TG TFTs (TFTs without physical bottom gate) shows poor TFT characteristics. The typical transfer and output characteristics of these TFTs $(W / L=15 / 5 \mu \mathrm{m} / \mu \mathrm{m})$ are shown in Fig. $5(\mathrm{a})$ and $5 \mathrm{~b}$, respectively. The microscopic image of these TFTs is also shown in Fig. 5(a) (inset view). The characteristics are also listed in Table. 1. It can be seen that a very negative $\mathrm{V}_{\mathrm{ON}}$ (approximately $-6.0 \mathrm{~V}$ ) and poor $\mathrm{SS}^{-1}$ is observed for these TFTs. In output characteristics, similar to DG TFTs, no sshaped behavior is observed at low values of $\mathrm{V}_{\mathrm{DS}}$; however, the output resistance $\left(\Delta \mathrm{I}_{\mathrm{DS}} / \Delta \mathrm{V}_{\mathrm{DS}}\right)$ is high in comparison with the DG TFTs. This is possibly due to poor shielding of
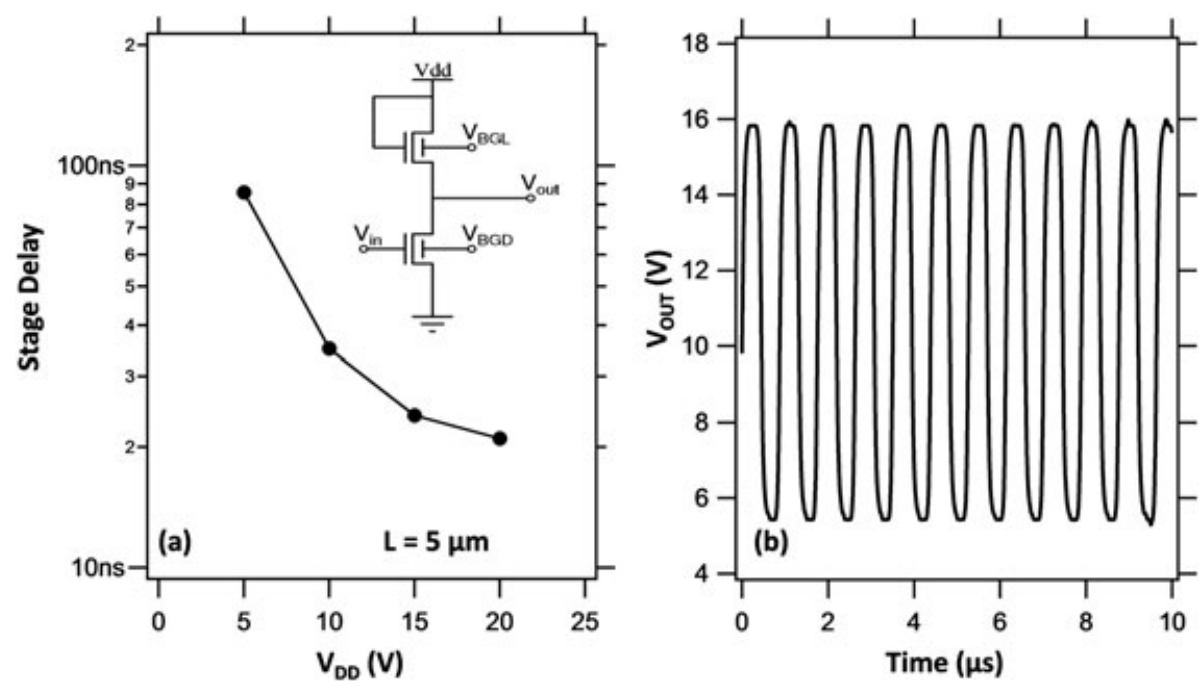

FIGURE 8 - (a) Stage delay versus supply voltage for dual-gate 19-stage ring oscillators, designed in diode-load logic for $L=5 \mu \mathrm{m}$ (inset; inverter scheme used in this work, whereby $V_{B G L}$ and $V_{B G D}$ are independent biases); (b) output pattern of dual-gate oscillator at $\mathrm{V}_{\mathrm{DD}}=20 \mathrm{~V}$. 
channels from the bottom, a possible impact of buffer layer out-diffusion happened during the final annealing step. ${ }^{27} \mathrm{In}$ Fig. 6, the statistical plots of $\mathrm{V}_{\mathrm{ON}}$ and $\mathrm{I}_{\mathrm{ON}}$ of $100 \mathrm{DG}$ control TFTs (at $\mathrm{V}_{\mathrm{DS}}=10 \mathrm{~V}$ ) is compared with isolated TG TFTs. It is observed that both $\mathrm{V}_{\mathrm{ON}}$ and $\mathrm{I}_{\mathrm{ON}}$ spread is better for DG control TFTs in comparison with isolated TG TFTs. In another TFT characterization, the bias-stress stability of DG (under different gate control) and isolated TG TFTs with an identical a-IGZO channel is investigated. All the TFTs were stressed at room temperature under $+1 \mathrm{MV} / \mathrm{cm}\left(\mathrm{V}_{\mathrm{GS}}-\mathrm{V}_{\mathrm{ON}}=+20 \mathrm{~V}\right.$ and $\left.\mathrm{V}_{\mathrm{DS}}=0 \mathrm{~V}\right)$ in positive direction and at $-1 \mathrm{MV} / \mathrm{cm}\left(\mathrm{V}_{\mathrm{GS}^{-}}\right.$ $\mathrm{V}_{\mathrm{ON}}=-20 \mathrm{~V}$ and $\left.\mathrm{V}_{\mathrm{DS}}=0 \mathrm{~V}\right)$ in negative direction for a period of $10^{4} \mathrm{~s}$. It is observed that the mobility (linear), $\mathrm{SS}^{-1}$, and $\mathrm{I}_{\mathrm{ON}} /$ $\mathrm{I}_{\mathrm{OFF}}$ ratio are quite similar before and after the complete stressing experiment for all the TFTs. However, as shown in Fig. 7 , a lower threshold voltage $\left(\mathrm{V}_{\mathrm{TH}}\right)$ change of less than $1.0 \mathrm{~V}$ (in both positive and negative directions) is observed for the DG TFTs in comparison with isolated TG TFTs. These good results for the DG control TFTs could also be due to the presence of the additional metallic bottom gate that provides better encapsulation to the channel ${ }^{28-32}$

In circuits, the realization of ring oscillator (19-stage) will provide a reliable estimate of the speed of the logic gates that can be made in this technology. ${ }^{14,15,19,25,29,33-35}$ The inverter schematic that allows the independent biasing of $\mathrm{V}_{\mathrm{BGL}}$ (BG load) and $\mathrm{V}_{\mathrm{BGD}}$ (BG drive) is depicted in the inset of Fig. 8 (a). ${ }^{33}$ The resulting stage delay versus the supply voltage of the ring oscillators is plotted in Fig. 8(a). At each measurement point, we have used specific bias voltages for the back gates to obtain the maximum frequency or minimum delay. As an example, at a supply voltage of $20 \mathrm{~V}$, we applied $\mathrm{V}_{\mathrm{BGD}}$ of $6 \mathrm{~V}$ and $\mathrm{V}_{\mathrm{BGL}}$ of $35 \mathrm{~V}$, which resulted in an oscillation frequency of $1.24 \mathrm{MHz}$. This corresponds to a stage delay of $20 \mathrm{~ns}$. The voltage output curve of the oscillator at a voltage drain drain $\left(\mathrm{V}_{\mathrm{DD}}\right)$ of $20 \mathrm{~V}$ is shown in Fig. $8(\mathrm{~b})$.

\section{Conclusion}

In summary, the DG a-IGZO TFTs with improved performance and lesser mask steps have been demonstrated. It has been shown that with the DG control, the TFTs have higher $\mathrm{I}_{\mathrm{ON}}$, steeper $\mathrm{SS}^{-1}$, lower output resistance, $\mathrm{V}_{\mathrm{ON}}$ closer to zero volts, and smaller $\mathrm{V}_{\mathrm{TH}}$ shift under bias stress in comparison with TFTs under single-gate control. We also demonstrated the applicability of these TFTs in logic circuitry.

\section{Acknowledgment}

This work was performed in a collaboration between imec and TNO in the frame of the HOLST Centre.

\section{References}

1 K. Nomura et al., "Amorphous oxide semiconductors for high-performance flexible thin-film transistors," Nature 488, 432, 488-492 (2004).
2 J. S. Park et al., "Review of recent developments in amorphous oxide semiconductor thin film transistor devices," Thin Solid Films, 520, No. 6, 1679-1693 (2012).

3 P. Barquinha et al., "Toward high-performance amorphous IGZO TFTs," J. Electrochem. Soc., 156, No. 3, H161-H168 (2009).

4 M. Nag et al., "Single-source dual-layer amorphous IGZO thin-film transistors for display and circuit applications," J. Soc. Inf. Disp., 21, No. 3, 129-136, Mar. (2013).

5 M. Nag et al., "Novel backchannel-etch process flow based a-IGZO TFTs for circuit and display applications on PEN-foil," J. Soc. Inf. Disp., 21, No. 9, 369-375, Sep. (2013).

6 M. Nag et al., "High performance a-IGZO thin-film-transistors with mfPVD $\mathrm{SiO}_{2}$ as an etch-stop-layer," J. Soc. Inf. Disp., 22, No. 1, 23-28, January (2014).

7 A. D. Mourey et al, "Self-aligned-gate ZnO TFT circuits," IEEE Electron Device Letters, 31, No. 4, 326-328, April (2010).

8 N. Morosawa, "A novel self-aligned top-gate oxide TFT for AM-OLED displays” Sony Corporation, 479482, SID 2011.

9 S. Kim et al., "Source/drain formation of self-aligned top-gate amorphous GaInZnO thin-film transistors by $\mathrm{NH}_{3}$ plasma treatment", IEEE Electron Device Letters, Vol. 30, No. 4, April 2009, p. 374-376.

10 B. D. Ahn et al., "Comparison of the effects of $\mathrm{Ar}$ and $\mathrm{H}_{2}$ plasmas on the performance of homo junctioned amorphous indium gallium zinc oxide thin film transistors," Appl. Phys. Lett., 93, No. 20, 203-506, Nov. (2008)

11 J. Park et al., "Self-aligned top-gate amorphous gallium indium zinc oxide thin film transistors," Appl. Phys. Lett., 93, No. 5, 053501, Aug. (2008).

12 C. H. Wu et al., "Self-aligned top-gate coplanar In-Ga-Zn-O thin-film transistors," J. Display Technol., 5, No. 12, 515-519, Dec. (2009).

13 D. H. Kang et al., "Self-aligned coplanar a-IGZO TFTs and application to high-speed circuits," IEEE Electron Device Lett., 32, No. 10, 1385-1387, Oct. (2011).

14 M. Nag et al., "Circuits and AMOLED display with self-aligned a-IGZO TFTs on polyimide foil," J. Soc. Inf. Disp., 22, No. 10, 509-517, October (2014).

15 S. Steudel et al., "Flexible AMOLED display with integrated gate driver operating at operation speed compatible with 4k2k", SID Symposium Digest of Technical Papers, 46, 2015.

$16 \mathrm{~K}$. -S. Son et al., "Highly stable double-gate Ga-In-Zn-O thin-film transistor," IEEE Electron Device Lett., 31, No. 8, 812-814, Aug. (2010).

17 G. Baek et al., "Electrical properties and stability of dual-gate coplanar homojunction DC sputtered amorphous indium-gallium-zinc-oxide thinfilm transistors and its application to AM-OLEDs," IEEE Trans. Electron Devices, 58, No. 12, 4344-4353, Dec. (2011).

18 M. Mativenga et al, "Bulk accumulation a-IGZO TFT for high current and turn-on voltage uniformity," IEEE Electron Device Lett., 34, No. 12, 1533-1535, Dec. (2013).

19 M. J. Seok et al., "A full-swing a-IGZO TFT-based inverter with a topgate-bias-induced depletion load," IEEE Electron Device Lett., 32, No. 8, 1089-1091, Aug. (2011).

$20 \mathrm{~S}$. Zhang et al., "A novel self-aligned double-gate TFT technology," IEEE Electron Device Lett., 22, No. 11, 530-532, Nov. (2001).

21 N. Münzenrieder et al., "Flexible self-aligned double-gate IGZO TFT," IEEE Electron Device Lett., 35, No. 1, 69-71, Jan. (2014).

22 J.-G. Yun et al., "Independent double-gate fin SONOS flash memory fabricated with sidewall spacer patterning," IEEE Trans. Electron Devices, 56, No. 8, 1721-1728, Aug. (2009).

$23 \mathrm{X}$. Li et al., "High-speed dual-gate a-IGZO TFT-based circuits with topgate offset structure," IEEE Electron Device Letters, 35, No. 4, 461, April (2014).

24 N. Münzenrieder et al., "Flexible double gate a-IGZO TFT fabricated on free standing polyimide foil," Solid-State Electron., 84, 198-204, Jun. (2013).

25 N. Münzenrieder et al., "Flexible self-aligned amorphous InGaZnO thinfilm transistors with sub-micrometer channel length and a transit frequency of $135 \mathrm{MHz}$, IEEE Trans. Electron Devices, 60, No. 9, 2815-2820, Sep. (2013).

26 F. M. Li et al., "Flexible barrier technology for enabling rollable AMOLED displays and upscaling flexible OLED lighting," SID Symposium Digest of Technical Papers, 44, No. 1, 199-202, June (2013).

27 M. Nag et al., "Impact of buffer layers on the self-aligned top-gate a-IGZO TFT characteristics," SID Symposium Digest of Technical Papers, Issue 1 , 46, No. 1, 1139-1142, June (2015). 
$28 \mathrm{X}$. He et al., "Implementation of fully self-aligned homojunction doublegate a-IGZO TFTs," IEEE Electron Device Letters, 35, No. 9, September (2014).

29 M. Nag et al., "Dual-gate self-aligned a-IGZO TFTs using 5-mask steps," IDW-2015, Otsu, Japan

$30 \mathrm{G}$. Baek et al., "Electrical instability of double-gate a-IGZO TFTs with metal source/drain recessed electrodes," IEEE Transactions On Electron Devices, 61, No. 4, 1109, April (2014).

$31 \mathrm{~K}$. -J. Chang et al., "a-IGZO TFTs reliability improvement by dual gate structure," pp. 1023, SID 2015 DIGEST.

$32 \mathrm{X}$. Li et al., "Effect of bulk-accumulation on switching speed of dual-gate a-IGZO TFT-based circuits," IEEE Electron Device Letters, 35, No. 12 1242, December (2014).

$33 \mathrm{~K}$. Myny et al., "Unipolar organic transistor circuits made robust by dualgate technology," IEEE Journal of Solid-State Circuits, 46, No. 5, 1223, May (2011).

34 R. E. Presley et al., "Transparent ring oscillator based on indium gallium oxide thin-film transistors," Solid-State Electron., 50, 500-503 (2006).

35 A. K. Tripathi et al., "Low-voltage gallium-indium-zincoxide thin film transistors based logic circuits on thin plastic foil: building blocks for radio frequency identification application," Appl. Phys. Lett., 98, 162102 (2011).

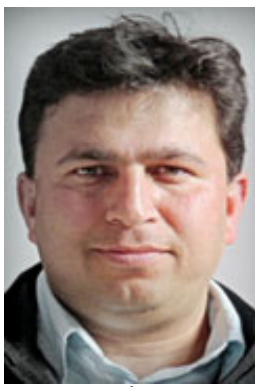

Manoj Nag received his Master of Technology degree in December, 1998 in Solid State Materials from the Indian Institute of Technology, Delhi, India. After that, he worked as a Process Engineer with STMicroelectronics Singapore from January, 2000 to June, 2005. From July, 2005 to September, 2007, he was working with International Rectifier (Now Infineon) in UK as a Snr Process Engineer. From October, 2007 to May, 2009, he was with Qimonda in Germany as a Technology Transfer Module Leader. At these places, he was involved with process integration and characterization of power and memory ICs. He joined imec as a Snr Process Development Engineer in June, 2009. He also finished his PhD degree entitled "Study of Process Technology and Device Architecture for Amorphous Indium Gallium Zinc Oxide Thin Film Transistors" in December, 2017 under the guidance of Prof. Paul Heremans and Prof. Guido Groeseneken at IMEC and Katholieke Universiteit Leuven (KUL), Belgium. His research interest includes a-IGZO TFTs for display backplanes and thin-film circuitry applications.

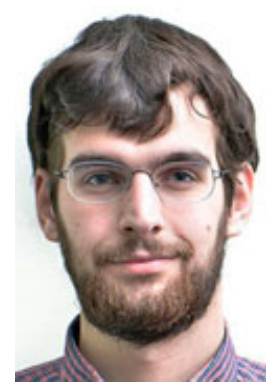

Florian De Roose was born in Sint-Niklaas, Belgium, in 1990. In 2013, he graduated and received his MSc in Electrical Engineering, Electronics and Integrated Circuits. The subject of his MS thesis was the design of an accurate frequency reference based on transmission lines. $\mathrm{He}$ is currently working as a research assistant under the guidance of Prof. Dr. Ir. W. Dehaene and Prof. Dr. Ir. J. Genoe at the MICAS laboratories of the KU Leuven and the LAE department of imec towards a PhD degree on the design of advanced mixed mode circuits in oxide technologies.

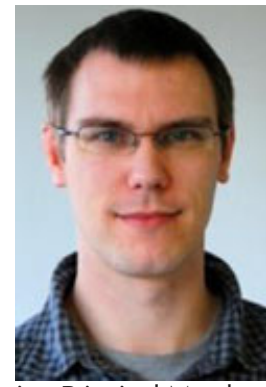

Kris Myny was born in Hasselt, Belgium, on July 26, 1980. He received the master degree at the Katholieke Hogeschool Limburg in Diepenbeek, Belgium, in 2002. He joined imec in Leuven in 2004 as a member of the Polymer and Molecular Electronics group. In 2013, he recieved the PhD degree on the design of organic circuits. His main research interests are the design, fabrication, and optimization of digital organic circuits for amongst others organic RFID tags and AMOLED backplanes. He received the IMEC 2010 Scientific Excellence Award and the 2011-2012 IEEE SSCS Predoctoral Achievement Award. Currently, he is a Principal Member of Technical Staff at imec.

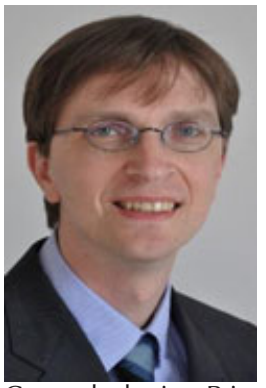

Soeren Steudel received the MSc degree in Electrical Engineering from the Dresden, University of Technology in Germany and a PhD degree from the Katholieke Universiteit Leuven, Belgium, in 2002 and 2007, respectively. He is working since 2002 at imec on TFTs and rectifying diodes. This work resulted in more than 20 journal publications, several patents, and numerous conference contributions. His research interest includes flexible backplanes for display application and process integration of organic and metal oxide semiconductor and thin film circuits on foil. Currently, he is a Principle Member of Technical Staff at IMEC.

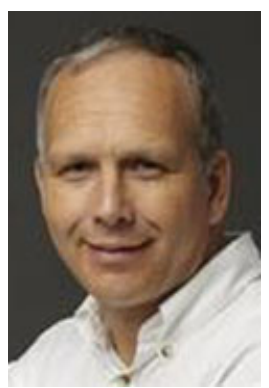

Jan Genoe was born in Leuven, Belgium, on May 19, 1965. He received the MS degree in Electrical Engineering and the $\mathrm{PhD}$ degree from the Katholieke Universiteit Leuven, in 1988 and 1994, respectively. Afterward, he joined the Grenoble High Magnetic Field Laboratory as a Human Capital and Mobility Fellow of the European Community. In 1997, he became a Lecturer at the Katholieke Hogeschool Limburg (KHLim) in Diepenbeek, Belgium. Currently, he is both a professor at the Katholieke Universiteit Leuven, Belgium, and a chief Scientist at imec. His current research interests are organic and oxide transistors and circuits as well as organic photovoltaics. He is the author and coauthor of about 90 papers in refereed journals. Jan Genoe is a member of the IEEE.

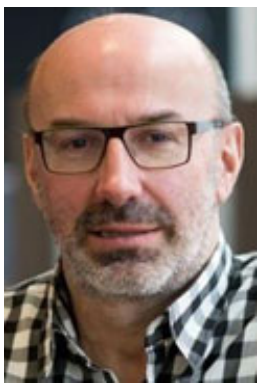

Guido Groeseneken received the MSc degree in Electrical and Mechanical Engineering (1980) and the PhD degree in Applied Sciences (1986), both from the Katholieke Universiteit Leuven, Belgium. In 1987, he joined the R\&D Laboratory of IMEC (Interuniversity Microelectronics Center) in Leuven, Belgium, where he is responsible for research in reliability physics for deep submicron CMOS technologies. From October 2005 to April 2007, he was also responsible for the imec Post CMOS Nanotechnology program within imec's core partner research program. He became an IEEE Fellow in 2005 and an IMEC Fellow in 2007.

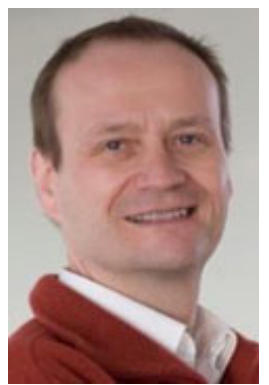

Paul Heremans received the $\mathrm{PhD}$ degree in Electrical Engineering in 1990 at the University of Leuven, Belgium, on hot-carrier degradation of MOS transistors. He then joined the optoelectronics group of IMEC, where he worked on optical interchip interconnects, and on highefficiency III-V thin-film surface-textured lightemitting diodes. His current research interest is oxide and organic electronics, including circuits, backplanes, and memories, as well as organic photovoltaics. He is an imec Fellow, a Director of IMEC's Large Area Electronics department, and a part-time Professor at the Electrical Engineering Department of the University of Leuven. 